\title{
The Use of a High-Resolution, High-Contrast X-ray Microscope to Probe the Internal Structure of Low Z Materials
}

\author{
Joseph D. Ferrara ${ }^{1}$, Yoichi Araki ${ }^{2}$, Kensaku Hamada $^{2}$, Kazuhiko Omote $^{2}$, Yoshihiro Takeda ${ }^{2}$ \\ 1. Rigaku Americas Corporation, X-ray Research Laboratory, The Woodlands, Texas, USA \\ 2. Rigaku Corporation, X-ray Research Laboratory, Akishima-shi, Tokyo, Japan
}

We have developed an X-ray microscope that incorporates a high-intensity, exchangeable target X-ray source, a high-precision rotation stage, and a high-resolution X-ray detector. Figure 1 displays a schematic of the essential components of the system. The X-ray generator is of the rotating anode type with a focal spot size of $70 \mu \mathrm{m}$, operating at a maximum power of $1200 \mathrm{~W}$. The anodes are swappable, which allows us to optimize the wavelength to the particular sample under investigation, maximizing contrast or minimizing absorption. Anode materials for the X-ray source include chromium, copper and molybdenum. In Figure 2, we show transmission images collected on a sample of plastic foam. In this case, $\mathrm{Cr}$ radiation provides the best contrast for this particular low molecular weight (low Z) material.

The high-resolution X-ray detector consists of a scintillator, a lens and a cooled CCD detector. The lens is swappable, providing for a field of view (FOV) as large as $14.4 \mathrm{~mm} \times 10 \mathrm{~mm}$ at $4.32 \mu \mathrm{m} /$ pixel. Highresolution mode is achievable through the use of a lens with a FOV of $0.9 \mathrm{~mm} \times 0.7 \mathrm{~mm}$ at 0.270 $\mu \mathrm{m} /$ pixel. With this configuration we are able to resolve two-dimensional features to $0.6 \mu \mathrm{m}$ and threedimensional features to $0.8 \mu \mathrm{m}$.

Figure 3 demonstrates one experiment in which an over-the-counter tablet was analyzed with Mo radiation. Then a single particle representing the active pharmaceutical ingredient (API) was extracted and analyzed with $\mathrm{Cr}$ radiation. In both experiments, complete computed tomography (CT) data sets were collected and the images reconstructed for the analysis using software developed in our lab. In the Mo experiment, the tablet was found to have a mean particle size distribution of diameter $213 \mu \mathrm{m}$ with a maximum value of $350 \mu \mathrm{m}$ and minimum of $190 \mu \mathrm{m}$. In the $\mathrm{Cr}$ experiment, the single particle was found to consist of several layers, with the innermost displaying cracks and voids. The single particle was not damaged in the experiment, so it is available for other experiments to test the efficacy of the API in this formulation.

Further results will be presented on other light material samples, such food products, carbon fiber reinforced polymers and composites. 


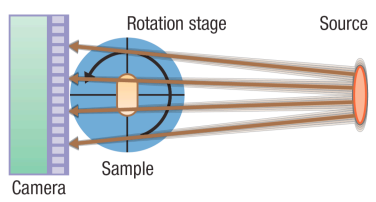

Figure 1. Schematic representation of the optical geometry of the instrument. The source can be $\mathrm{Cr}, \mathrm{Cu}$ or Mo. The pixel size at the detector ranges from $4.32 \mu \mathrm{m}$ to $0.270 \mu \mathrm{m}$.

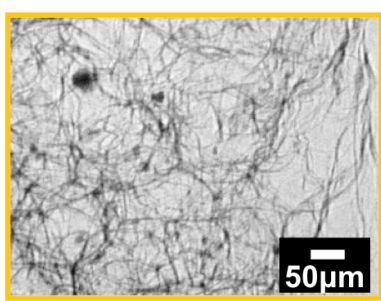

Cr source, $5.4 \mathrm{keV}$

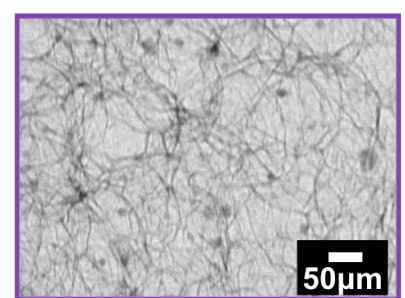

Cu source, $8 \mathrm{keV}$

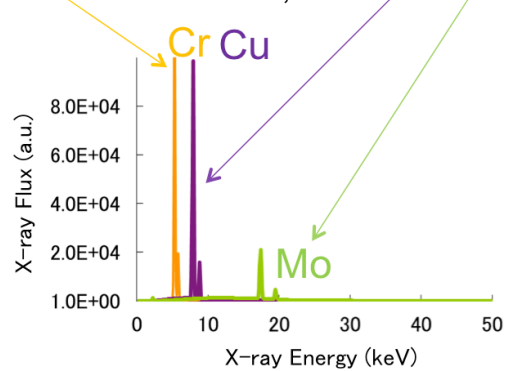

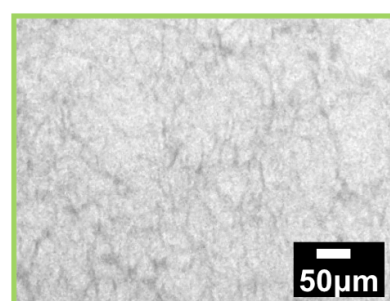

Mo source $17 \mathrm{keV}$

Figure 2. Comparison of the contrast for a low $\mathrm{Z}$ foam using $\mathrm{Cr}, \mathrm{Cu}$ and $\mathrm{Mo}$ radiation. In this experiment only the target material in the anode has been changed.

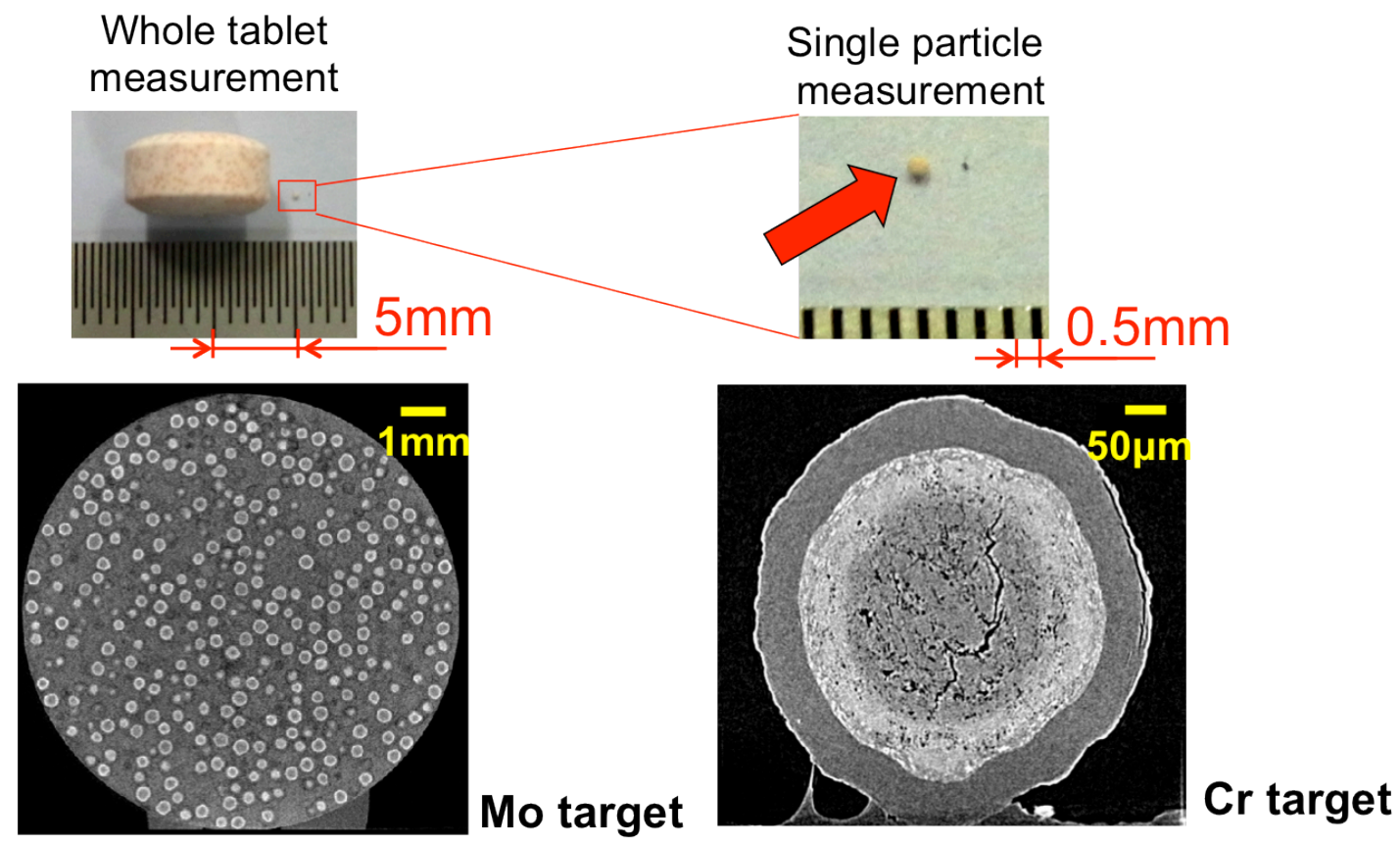

Figure 3. Whole tablet and single particle analysis of an over-the-counter pharmaceutical demonstrating the results of selecting the appropriate wavelength for sample size and composition. 